\title{
Nidotherapy in the treatment of substance misuse, psychosis and personality disorder: secondary analysis of a controlled trial
}

\author{
Peter Tyrer, ${ }_{1}^{1}$ Katarina Milošeska, ${ }^{2}$ Craig Whittington, ${ }^{3}$ Maja Ranger, ${ }^{2}$ Ibrahim Khaleel, ${ }^{4}$ \\ Mike Crawford, ${ }^{1}$ Bernard North, ${ }^{5}$ Barbara Barrett ${ }^{6}$
}

The Psychiatrist (2011), 35, 9-14, doi: 10.1192/pb.bp.110.029983

\begin{abstract}
${ }^{1}$ Imperial College London, UK; ${ }^{2}$ London Deanery; ${ }^{3}$ National Collaborating Centre for Mental Health, Centre for Outcomes Research and Effectiveness, University College London; ${ }^{4}$ Mahatma Gandhi Institute of Comprehensive Mental Healthcare, Guntur, India; ${ }^{5}$ Statistical Advisory Service, Imperial College London; ${ }^{6}$ Institute of Psychiatry, London, UK

Correspondence to Peter Tyrer (p.tyrer@imperial.ac.uk)
\end{abstract}

\begin{abstract}
Aims and method To examine the clinical outcome and bed usage in patients with comorbid substance misuse and psychosis. The patients were randomised to ordinary assertive outreach team care or to enhanced assertive outreach with nidotherapy. Ratings of clinical symptoms, social function, engagement with services, bed usage (primary outcome after 1 year) and economic costs were assessed at baseline and at 6 and 12 months after randomisation.
\end{abstract}

Results Patients referred to nidotherapy had similar reduction in symptoms and engagement, with marginal superiority in social function $(P=0.045)$. There was a $110 \%$ reduction in hospital bed use after 1 year compared with control assertive care $(P=0.03)$. The mean cost savings for each patient allocated to nidotherapy was $£ 14705$ per year, mainly as a consequence of reduced psychiatric bed use.

Clinical implications Nidotherapy shows promise in the treatment of substance misuse and psychosis and may reduce hospital bed usage.

Declaration of interest P.T. has introduced nidotherapy to clinical practice and therefore has the natural bias of a product champion. No other author has any interests to declare.

\begin{abstract}
Nidotherapy (after nidus, 'nest') was first introduced as a treatment for people with complex personality disorders who had failed to respond to other treatment. ${ }^{1}$ It involves the systematic assessment and modification of the environment to help in minimising the impact of any form of mental disorder on the individual or on society. ${ }^{2,3}$ The treatment does not aim to treat the patient directly; it involves an environmental analysis of personal, social and physical environments, the setting of environmental targets, constructing a plan for environmental change (nidopathway), as well as monitoring the progress of this and making changes as necessary. ${ }^{4}$
\end{abstract}

A randomised controlled trial of nidotherapy was carried out in a population with patients in an assertive outreach team in which enhanced care with nidotherapy was compared with ordinary assertive outreach treatment alone. ${ }^{5}$ This included 52 patients with comorbid personality disorder and severe mental illness and showed nonsignificant reductions in bed usage, clinical symptoms and social functioning, but with sufficient reduction in cost to regard the treatment as cost-effective. These patients also included many with additional pathology, notably comorbid substance misuse, which in previous investigations ${ }^{6}$ has proved very difficult to treat, with no single approach having clear effectiveness over ordinary care. Because of this and because this group of patients accounts for many days of in-patient treatment, the outcome of 37 patients with substance misuse and psychosis who were randomised in the trial ${ }^{5}$ was felt to be of sufficient interest to carry out a secondary analysis of the data, and this is reported in this paper.

\section{Method}

The methodology of the study has been described elsewhere. ${ }^{5}$ It involved a simple parallel design with two treatment arms: nidotherapy-enhanced assertive treatment (referred to henceforth as nidotherapy group) and standard assertive outreach treatment (control group). In both the original study and our secondary analysis the main null hypothesis being tested was that nidotherapy and ordinary assertive outreach treatment would reduce the usage of hospital beds equally. The secondary hypotheses were that reduction of psychiatric symptoms and improvement in social functioning and engagement would also be similar in nidotherapy and control groups. These were a priori hypotheses for the main trial and transferred to the secondary analysis. No power calculation was made to establish the sample size and it was appreciated that 
definitive data were unlikely to be obtained from this small study.

\section{Patients}

The patients in this secondary study were all those diagnosed with substance dependence and a psychotic disorder recruited from the case-load of an assertive outreach and rehabilitation team in central London. The sample comprised 37 patients, randomised over 13 months, with 35 and 26 assessed at 6 and 12 months respectively, and with service usage data and costs on 34 patients. The diagnosis was derived from case notes using ICD-10 diagnoses and in equivocal cases the OPCRIT system ${ }^{7}$ was used to confirm substance dependence.

\section{Inclusion and exclusion criteria}

Patients were selected from the existing clientele in the assertive outreach team if they had severe mental illness and personality disorder. For the secondary analysis they needed to satisfy the diagnostic criteria for schizophrenia, schizoaffective disorder or bipolar disorder, together with dependence on amphetamines, opiates, cocaine, cannabis or alcohol. They were also required to give informed consent and were not in a stable phase at the time of recruitment (i.e. they were clinically unwell or unstable, despite receiving appropriate, evidence-based treatment). Exclusion criteria included inability to understand the questionnaires or procedures because of English language competence or intellectual disability, and age under 18. In addition to clinical assessment, patients also had their personality status assessed, using the ICD - 10 version of the Personality Assessment Schedule (PAS-I). ${ }^{8}$ Ethical approval was given by St Mary's Hospital ethical committee.

\section{Procedure}

After baseline assessment, those who satisfied the eligibility criteria and who were part of the case-load of the assertive outreach team between August 2003 and September 2004 were recruited. They were randomly allocated by an independent statistician, using a random numbers design with no stratification of groups, to either nidotherapy or control groups. Patients were assessed at baseline and at 6 and 12 months by M.R. and I.K., who remained blind of trial allocation; this was assisted by the fact that the nidotherapists in the team also worked as support workers with other patients and so their involvement did not disclose their therapeutic role.

\section{Assessments}

Assessments were made of:

- clinical psychopathology, using the Brief Psychiatric Rating Scale (BPRS) ${ }^{9}$

- social function, using key-worker and patient self-rated versions of the Social Functioning Questionnaire (SFQ) ${ }^{10}$

- in-patient bed usage, from an independent assessment of hospital record data

- engagement with services, using the Engagement and Assessment Scale (EAS) ${ }^{11}$

- all service costs, using the Secure Facilities Service Use Schedule (SFSUS), ${ }^{12}$ which covers every possible service contact for those with severe mental illness in secure facilities and in the community.

\section{Primary and secondary outcomes}

The duration of psychiatric admissions after 1 year was selected in advance as the primary outcome because the positive value of nidotherapy is judged by its ability to keep patients in a harmonious relationship with their home environment. All other outcomes were secondary and tested at 6 months and 1 year.

\section{Interventions}

\section{Nidotherapy group}

Those allocated to nidotherapy-enhanced assertive treatment received up to 15 sessions of nidotherapy, of variable length, from two nidotherapists following a standard format. $^{3}$ This involved a combination of environmental analysis, articulation of the patient's needs at a physical, social and personal environmental level, and setting of targets. A flexible approach was used in the timing and frequency of sessions as the plan created (nidopathway) is determined by both therapist and patient. Where changes suggested by the nidotherapist conflicted with those of the clinical team, discussion followed and an agreed consensus was reached. In some instances, no major changes were suggested in the environment, but adjustments were made to suit patient preferences. Patients in this group continued to be seen by the assertive outreach team and no treatment approach normally used by the team was denied them.

\section{Control group (ordinary assertive outreach)}

Patients allocated to this group received the standard outreach therapy of the team. (A previous randomised trial had established that this particular team was at least as effective as other assertive outreach teams. ${ }^{1}$ )

\section{Economic evaluation}

Costs were assessed from the perspective of service providers, including the health, social, voluntary and criminal justice services. The SFSUS was used to record service use during the 6 months immediately before randomisation and the 12 months after randomisation. All costs were calculated for the financial year 2004/2005. The cost of the nidotherapy was based on the time spent by the therapist interviewing the patient and reporting to the clinical team responsible for their care, plus relevant overheads. In order to calculate total costs, unit costs were applied to each service. Hospital services were costed using National Health Service reference costs, ${ }^{13}$ with published unit costs applied to community health and social services, ${ }^{14,15}$ medication ${ }^{16}$ and criminal justice services. ${ }^{17,18}$

\section{Statistical analysis}

All analyses were carried out on an intention-to-treat basis; analysis of covariance with adjustment for baseline differences was used with the rating scale data. To compare the differences in bed days occupied between the two groups, we used the method of Welsh \& Zhou $^{19}$ in 
combining the logistic and log-transformed multiple regressions into a common framework. To allow for baseline differences, we planned to calculate the average (original scale, unlogged) 12-month admission days for both treatment groups for a patient with an average number of baseline admissions and to provide confidence intervals for the two treatment means to allow an assessment of differences at 12 months.

\section{Clinical outcomes}

The clinical data measured on each occasion were analysed by repeated measures analysis of variance, with adjustment for baseline values, and analysis of data by last observation carried forward (LOCF) as well as case analysis of full data. Bed usage was recorded by measuring the number of days spent in hospital in the previous 6 months, adjusted for baseline (also the previous 6 months) and treatment group. The analysis of variance was run on SPSS 13 for Windows.

\section{Evaluation of costs}

Costs were compared between the nidotherapy group and the control group using standard $t$-tests, despite the skewed distribution of cost data. This method enables inferences to be made about the arithmetic mean. ${ }^{20}$ Nonparametric bootstrapping was used to assess the robustness of confidence intervals to non-normality of the cost distribution. $^{21}$

\section{Results}

Of 52 patients included in the trial, there were 37 with substance misuse: 19 were allocated to the nidotherapy group and 18 to the control group. With regard to ethnic background, 22 of the patients were White (59\%), 8 were
Black African or Caribbean (2\%) and 7 were from other ethnic background (19\%). These were similarly distributed, apart from African Caribbeans, who were disproportionately represented in the nidotherapy group ( 5 in nidotherapy, 1 in control). As far as substance misuse is concerned, 25 regularly used cannabis (68\%), 15 had alcohol dependence (41\%), 4 misused cocaine (11\%), 2 benzodiazepines (5\%), and 1 each misused antiparkinsonian drugs and amphetamines (3\%); 10 were dependent on more than one drug (27\%). All patients satisfied the criteria for at least one personality disorder so, in effect, this could be described as a 'triplediagnosis study,. ${ }^{8}$ One patient with polydrug use allocated to the control group died 2 months after randomisation. The data reported therefore relate to 36 patients, except with regard to hospital admissions. A CONSORT diagram shows the pathway of patients through the trial (Fig. 1).

\section{Primary outcome}

Data for all time points up to 12 months were available for 35 patients. There was a $110 \%$ difference in bed usage at one year (nidotherapy group reduction 2\%; control group increase $108 \%$; $P=0.035$ ). However, the variance in the data was considerable. As bed usage increased in the control group with only a small reduction of bed usage in the nidotherapy group, the data illustrate the increasing problem of hospital bed use in those with dual diagnosis (substance misuse and psychosis).

\section{Secondary outcomes}

There were no meaningful differences between the outcomes in each group for clinical symptomatology and engagement, but also no evidence of inferiority for those allocated to nidotherapy. In the LOCF analysis there was

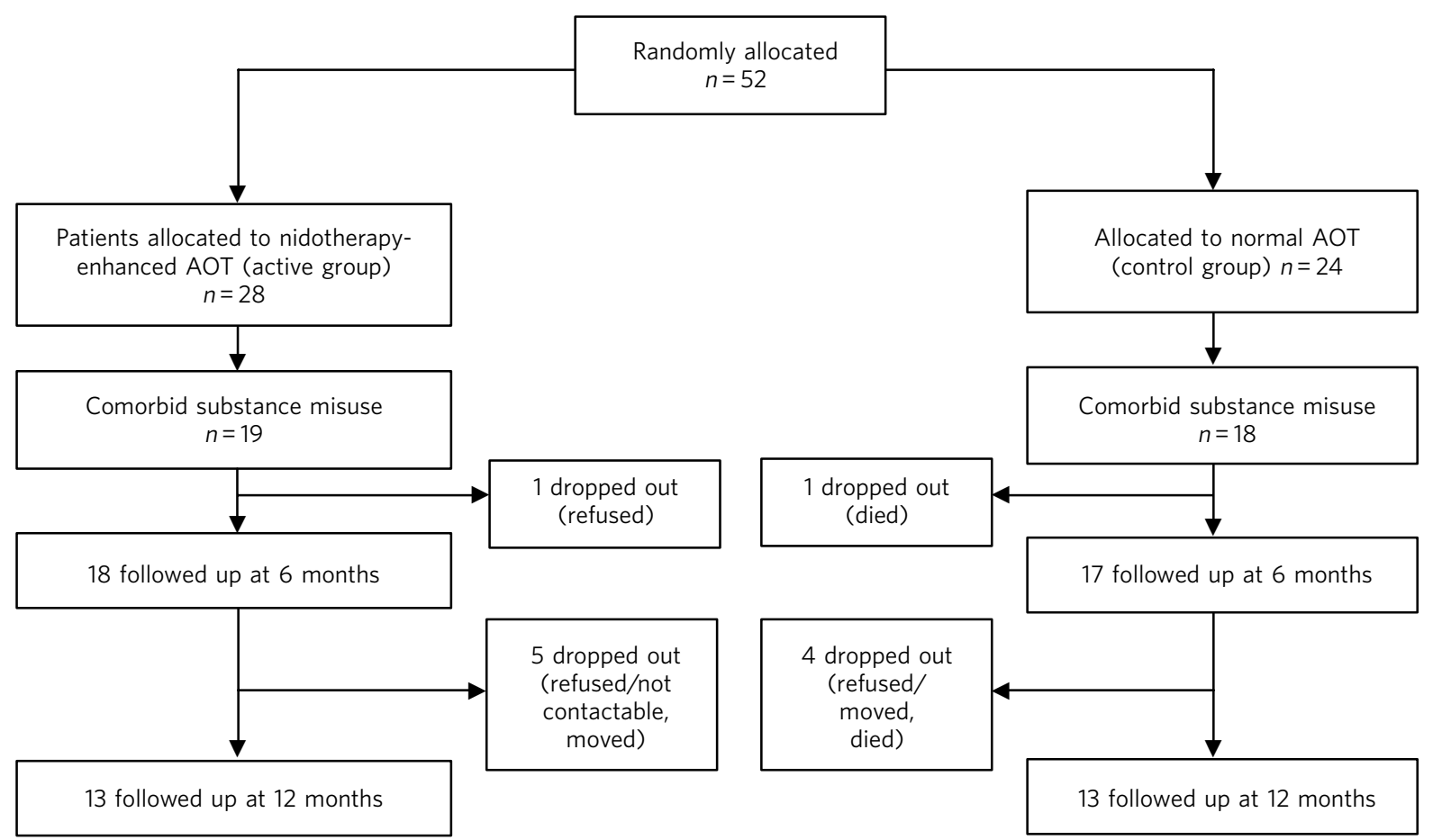

Fig 1 CONSORT diagram of selection of patients and their pathway through the trial. AOT, assertive outreach treatment. 
also greater improvement in social functioning in those allocated to nidotherapy $(P=0.045)$ (Table 1$)$.

Together, these findings support the view that nidotherapy did not achieve its reduction in bed use at the cost of adverse clinical outcomes. Service use data were available for 34 study participants with substance misuse, 19 in the nidotherapy group and 15 in the control group; of the three participants for whom service use data were not available, two individuals had left the country and one person died. Average total costs are detailed in Table 2 and follow a similar pattern to those seen in the main analysis, ${ }^{5}$ although the differences in costs are more pronounced, with an increase in bed usage in those in the control group. Over the 12 month follow-up total costs were $£ 14705$ less in the nidotherapy group compared with the treatment as usual group and this difference in cost was significant at the $10 \%$ level $(P=0.072)$. The difference in costs was primarily due to the substantial difference in the number of in-patient stays between the nidotherapy and treatment as usual groups, which is reflected in hospital costs of $£ 10938$ in the nidotherapy group compared with $£ 27871$ in the treatment as usual group. This was the main component behind the cost savings of $£ 14705$ per patient overall.

\section{Discussion}

The findings with this substantial subpopulation recruited to the randomised trial were quite surprising. The original trial of patients with severe mental illness and personality disorder showed some evidence of benefit and cost savings, ${ }^{5}$ but these were magnified greatly in this smaller population.
A trial of 37 patients can in no way be regarded as definitive, but the substantial impact of nidotherapy on bed usage was responsible for considerable cost savings that could be of clinical and economic importance. As patients with comorbid substance misuse and psychosis tend to remain in hospital for long periods $\mathrm{s}^{22,23}$ and are generally resistant to complex interventions, ${ }^{24}$ any treatment strategies that can reduce bed usage without impairing clinical outcomes are valuable.

The outcomes in this study suggest that there was no clinical handicap associated with addition of nidotherapy to the treatment programme and that social functioning, one of the main aims of nidotherapy, ${ }^{2}$ also improved. Engagement with services was not improved by nidotherapy, but as good engagement is one of the outcomes of assertive outreach that is superior to that of conventional community mental health teams, ${ }^{25}$ this finding is not unexpected. The possible difficulties in the separation of the nidotherapist from the main clinical services could have an impact on engagement. $^{26}$

\section{Limitations}

Despite these results, the study suffers from several limitations that reduce the strength of its conclusions. The main study hypothesis was not concerned with substance misuse and psychosis and this paper describes a secondary analysis only. The numbers are too small to reach any definitive conclusions and the drop-out rate of nearly $30 \%$ for the clinical data collection over the latter half of the 12-month period is too great to have confidence in the results of these measures. However, the independent

Table 1 Results of the secondary outcomes of clinical symptomatology (BPRS), social functioning (SFQ-KW) and
engagement with services (EAS) in nidotherapy and control groups for participants with substance misuse
problems at baseline

BPRS, Brief Psychiatric Rating Scale; EAS, Engagement and Acceptance Scale; LOCF, last observation carried forward; SFQ-KW, Social Functioning Questionnaire KeyWorker version.

${ }^{\star} P<0.05$ 


\begin{tabular}{|c|c|c|c|}
\hline & $\begin{array}{l}\text { Nidotherapy group, } n=19 \\
\text { Mean (s.d.) }\end{array}$ & $\begin{array}{c}\text { Control group, } n=15 \\
\text { Mean (s.d.) }\end{array}$ & $\begin{array}{c}\text { Mean } \\
\text { difference }\end{array}$ \\
\hline In-patient stays, nights & $54(75)$ & $139(135)$ & -85 \\
\hline $\begin{array}{l}\text { Healthcare }^{a} \\
\text { Hospital costs }{ }^{b} \\
\text { Community health services } \\
\text { Medication }\end{array}$ & $\begin{array}{c}15173(15786) \\
10938(14990) \\
3159(1628) \\
1076(1704) \\
\end{array}$ & $\begin{array}{c}31105(27290) \\
27871(26986) \\
2065(1256) \\
1170(875) \\
\end{array}$ & $\begin{array}{c}-15932 \\
-16932 \\
1094 \\
-94 \\
\end{array}$ \\
\hline $\begin{array}{l}\text { Social and voluntary services } \\
\text { Community } \\
\text { Accommodation }\end{array}$ & $\begin{array}{l}3559(5622) \\
1101(1288) \\
2458(5628)\end{array}$ & $\begin{aligned} & 2561(4466) \\
& 717(682) \\
& 1844(4618) \\
&\end{aligned}$ & $\begin{array}{l}998 \\
383 \\
615 \\
\end{array}$ \\
\hline Criminal justice & $181(787)$ & $2(7)$ & 179 \\
\hline Total costs & $18963(19010)$ & $33668(27022)$ & $-14705^{c}$ \\
\hline
\end{tabular}

a. All costs are given in British pounds

b. Hospital costs include all aspects of hospital treatment, including bed occupation

c. $95 \% \mathrm{Cl}-30791$ to $1380 ; P=0.072$

collection of the economic data had only $8 \%$ of patients unaccounted for, so more reliance can be placed on the differences in these data. We also have reasonable confidence that the research assessors were blind to the allocation of patients when recording their data because the nidotherapists involved in the study also worked with the patients in the control groups as support workers.

It is difficult to determine where nidotherapy might have had particular value in this population. Since it is concerned with getting a better environmental fit for the patient, it could promote more appropriate placements for patients after discharge. The role of the nidotherapist in acting as an advocate for the patient ${ }^{26}$ may also be important here, as the ability to get over personal wishes and needs to the clinical team in a coherent and consistent way was useful in effecting change. The wish most fervently expressed by the patients allocated to nidotherapy was that for greater autonomy and this was also taken account of in planning the nidotherapy strategy. One recurring theme in the patients with substance misuse was the wish to have greater personal control and not be persistently asked to stop misusing substances. In the practice of nidotherapy the emphasis on achieving a harmonious environmental fit quite independently of clinical pathology ${ }^{4,27}$ may be relevant here; individuals begin to notice that substance misuse may spoil the environmental fit and they may modify it accordingly without any external pressures.

The practice of nidotherapy is a complex process still undergoing development. Practitioners need to be skilled in accepting patients for what they are and not what they would like them to be, to work with them in a collaborative, non-judgemental way, and to be sensitive in separating unrealistic wants from genuine needs when deciding on an environmental change. All nidotherapists who have been trained to date have been independent practitioners, with skills that are primarily those of engagement and encouragement (our two nidotherapists were also acting as support workers in this study and had no specific mental health qualifications). This may have been an asset with a population that is generally sceptical of any psychiatric intervention because of past experiences. ${ }^{4}$ The additional role of advocate is also much appreciated in this group ${ }^{26}$ and, by taking every aspect of the patients' wishes seriously and considerately, fits in well with the promotion of recovery. The fact that not only bed usage was reduced in this study but community placements were made relatively cheaply (Table 2) suggests that the role of nidotherapist in 'customising' accommodation so that it makes a better fit for the patient is cost-effective. The possibility that this could bring wider benefit needs to be extended further in definitive randomised trials.

\section{Funding}

The study was supported by a grant from the Nicola Pigott Fund. International Trial no.: ISRCTN 73227556.

\section{Acknowledgements}

We thank Kofi Kramo, Andrew MacDonald, Sandra O'Sullivan, Nancy Ababio and Deirdre Dolan for their assistance in promoting the trial and ensuring that assessments were made blind of knowledge of allocation, Bharti Rao for acting as an independent randomiser of patients and Sandra O'Sullivan for administrative support throughout the trial.

\section{About the authors}

Peter Tyrer is Professor of Community Psychiatry, Centre for Mental Health, Imperial College London; Katarina Milošeska is CT3 in Psychiatry, London Deanery; Craig Whittington is Systematic Reviewer, National Collaborating Centre for Mental Health, Centre for Outcomes Research and Effectiveness, University College London; Maja Ranger is Consultant in Rehabilitation Psychiatry, Central and North West London Mental Health NHS Foundation Trust; Ibrahim Khaleel is Director, Mahatma Gandhi Institute of Comprehensive Mental Healthcare, Guntur, India; Mike Crawford is Reader in Mental Health Services Research, Imperial College London; Bernard North is Senior Statistician, Statistical Advisory Service, Imperial College London; Barbara Barrett is Lecturer, Centre for the Economics of Mental Health, Institute of Psychiatry, London, UK

\section{References}

1 Tyrer P. Nidotherapy: a new approach to the treatment of personality disorder. Acta Psychiat Scand 2002; 105: 469-71.

2 Tyrer P, Sensky T, Mitchard S. The principles of nidotherapy in the treatment of persistent mental and personality disorders. Psychother Psychosom 2003; 72: 350-6.

3 Tyrer P, Bajaj P. Nidotherapy: making the environment do the therapeutic work. Adv Psychiat Treat 2005; 11: 232-8. 
4 Tyrer P. Nidotherapy: Harmonising the Environment with the Patient. RCPsych Publications, 2009.

5 Ranger M, Tyrer P, Milošeska K, Fourie H, Khaleel I, North B, et al. Costeffectiveness of nidotherapy for comorbid personality disorder and severe mental illness: randomized controlled trial. Epidemiol Psichiatr Soc 2009; 18: 128-36.

6 Cleary M, Hunt G, Matheson S, Siegfried N, Walter G. Psychosocial interventions for people with both severe mental illness and substance misuse. Cochrane Database Syst Rev 2008; issue 1: CD001088.

7 McGuffin P, Farmer A, Harvey I. A polydiagnostic application of operational criteria in studies of psychotic illness. Development and reliability of the OPCRIT system. Arch Gen Psychiat 2001; 48: 764-70.

8 Tyrer P. Personality Assessment Schedule: PAS-I (ICD-10 version). In Personality Disorders: Diagnosis, Management and Course (2nd edn): 16080. Arnold, 2000

9 Overall JE, Gorman DL. The brief psychiatric rating scale. Psychol Rep 1962; 10: 799-812.

10 Tyrer $\mathrm{P}$, Nur U, Crawford M, Karlsen S, McLean C, Rao B, et al. The Social Functioning Questionnaire: a rapid and robust measure of perceived functioning. IntI J Soc Psychiat 2005; 51: 265-75.

11 Park MJ, Elsworth E, Fox J, Tyrer P, McDonald A. The Homelessness Engagement and Acceptance Scale (HEAS): a measure of engagement for the homeless mentally ill. Psychol Med 2002; 32: 855-61.

12 Barrett B, Byford S. Collecting service use data for economic evaluation in DSPD populations: Development of the Secure Facilities Service Use Schedule. Br J Psychiat 2007; 190 (suppl 49): s75-8.

13 Department of Health. NHS Reference Costs 2003-2004. Department of Health, 2005.

14 Curtis L, Netten A. Unit Costs of Health and Social Care. Canterbury Personal Social Services Research Unit, 2005.

15 Finn W, Hyslop J, Truman C. Mental Health, Multiple Needs and the Police. Revolving Doors Agency, 2000.
16 British Medical Association, Royal Pharmaceutical Society of Great Britain. British National Formulary 48. BMJ Books, Pharmaceutical Press, 2004

17 HM Prison Service. Prison Service Annual Report and Accounts. HM Prison Service, 2005

18 Legal Services Commission. General Criminal Contract. Legal Services Commission, 2003.

19 Welsh $\mathrm{AH}$, Zhou $\mathrm{XH}$. Estimating the retransformed mean in a heteroscedastic two-part model. J Statist Plan Infer 2006; 136: 860-81.

20 Thompson SG, Barber J. How should cost data in pragmatic randomised trials be analysed? BMJ 2000; 320: 1197-200.

21 Efron B, Tibshirani RJ. An Introduction to the Bootstrap. Chapman \& Hall, 1993.

22 Wade D, Harrigan S, Edwards J, Burgess PM, Whelan G, McGorry PD. Substance misuse in first-episode psychosis: 15-month prospective follow-up study. Br J Psychiatry 2006; 189: 229-34.

23 Keown P, Mercer G, Scott J. Retrospective analysis of hospital episode statistics, involuntary admissions under the Mental Health Act 1983 and number of psychiatric beds in England 1996-2006. BMJ 2008; 337: a1837.

24 Johnson S, Thornicroft G, Afuwape S, Leese M, White IR, Hughes E, et al. Effects of training community staff in interventions for substance misuse in dual diagnosis patients with psychosis (COMO study): Cluster randomised trial. Br J Psychiatry 2007; 191: 451-2.

25 Killaspy H, Bebbington P, Blizard R, Johnson S, Nolan F, Pilling S, et al. REACT: a Randomised Evaluation of Assertive Community Treatment in North London. BMJ 2006; 332: 815-9.

26 Spencer S-J, Rutter D, Tyrer P. Integration of nidotherapy into the management of mental illness and antisocial personality: a qualitative study. Int J Soc Psychiatry 2010; 56: 50-9.

27 Tyrer P, Kramo K. Nidotherapy in practice. J Ment Health 2007; 16: 117-31. 